\title{
On The Darboux Vector Belonging To Involute Curve A Different View
}

\author{
Süleyman ŞENYURT*, Yasin ALTUN and Ceyda CEVAHIR
}

(Communicated by Bülent ALTUNKAYA)

\begin{abstract}
In this paper, we investigated special Smarandache curves in terms of Sabban frame drawn on the surface of the sphere by the unit Darboux vector of involute curve. We created Sabban frame belonging to this curve. It was explained Smarandache curves position vector is composed by Sabban vectors belonging to this curve. Then, we calculated geodesic curvatures of this Smarandache curves. Found results were expressed depending on the base curve. We also gave example belonging to the results found.
\end{abstract}

Keywords: involute curve; Darboux vector; Smarandache curves; Sabban frame; geodesic curvature.

AMS Subject Classification (2010): 53A04.

${ }^{*}$ Corresponding author

\section{Introduction and Preliminaries}

The involute of the curve is well known by the mathematicians especially the differential geometry scientists. There are many essential consequences and properties of curves. Involute curves have been studied by some authors $[3,7]$. Whose position vector is composed by Frenet frame vectors regular curve is called a Smarandache curve [10]. Special Smarandache curves have been studied by some authors [1, 2, 5, 8, 9]. K. Taṣköprü, M. Tosun studied special Smarandache curves according to Sabban frame on $S^{2}$ [11]. Şenyurt and Çalişkan investigated special Smarandache curves in terms of Sabban frame of spherical indicatrix curves and they gave some characterization of Smarandache curves [4]. Let $\alpha: I \rightarrow E^{3}$ be a unit speed curve, we defined the quantities of the Frenet-Serret apparatus, respectively

$$
\begin{gathered}
T(s)=\alpha^{\prime}(s), \quad N(s)=\frac{\alpha^{\prime \prime}(s)}{\left\|\alpha^{\prime \prime}(s)\right\|}, B(s)=T(s) \wedge N(s), \\
\kappa(s)=\left\|T^{\prime}(s)\right\|, \quad \tau(s)=\left\langle N^{\prime}(s), B(s)\right\rangle .
\end{gathered}
$$

we have an orthonormal frame $\{T, N, B\}$ along $\alpha$. This frames is called the Frenet frame of $\alpha$. This curve the Frenet formulae are, respectively, [7]

$$
\left\{\begin{array}{l}
T^{\prime}(s)=\kappa(s) N(s) \\
N^{\prime}(s)=-\kappa(s) T(s)+\tau(s) B(s) \\
B^{\prime}(s)=-\tau(s) N(s) .
\end{array}\right.
$$

For any unit speed curve $\alpha: I \rightarrow \mathbb{E}^{3}$, the vector $\mathrm{W}$ is called Darboux vector defined by

Received : 11-June-2016, Accepted : 02-November-2016 


$$
W=\tau T+\kappa B
$$

If we consider the normalization of the Darboux, we have

$$
\sin \varphi=\frac{\tau}{\|W\|}, \quad \cos \varphi=\frac{\kappa}{\|W\|}
$$

and

$$
C=\sin \varphi T+\cos \varphi B
$$

where $\angle(W, B)=\varphi$, [6]. Let $\alpha: I \rightarrow \mathbb{E}^{3}$ unit speed and $\alpha^{*}: I \rightarrow \mathbb{E}^{3}$ be the $C^{2}$ - class differentiable two curves. If the tangent vector of the curve $\alpha$ is orthogonal to the tangent vector of the curve $\alpha^{*}$ which is called involute of the $\alpha$. According to definition, if the tangent of the curve $\alpha$ is denoted by $T$ and the tangent of the curve $\alpha^{*}$ is denoted by $T^{*}$, we can write [7]

$$
\left\langle T, T^{*}\right\rangle=0 .
$$

If the curve $\alpha^{*}$ is involute of $\alpha$, then we may write that [7]

$$
\alpha^{*}\left(s^{*}\right)=\alpha(s)+(c-s) T(s) .
$$

Let $\alpha: I \rightarrow E^{3}$ and $\alpha^{*}: I \rightarrow E^{3}$ be the $C^{2}$-class differentiable unit speed two curves and the amounts of $\{T(s), N(s), B(s)\}$ and $\left\{T^{*}\left(s^{*}\right), N^{*}\left(s^{*}\right), B^{*}\left(s^{*}\right)\right\}$ are entirely Frenet- Serret frame of the curves $\alpha$ and the involute $\alpha^{*}$, respectively, then [3]

$$
\left\{\begin{array}{l}
T^{*}=N \\
N^{*}=-\cos \varphi T+\sin \varphi B \\
B^{*}=\sin \varphi T+\cos \varphi B
\end{array}\right.
$$

where $\angle(W, B)=\varphi$. For the curvatures and the torsions we have

$$
\kappa^{*}=\frac{\|W\|}{|c-s| \kappa}, \quad \tau^{*}=\frac{\left(\kappa \tau^{\prime}-\tau \kappa^{\prime}\right)}{\kappa|c-s|\|W\|^{2}}
$$

where $\frac{d s^{*}}{d s}=|c-s| \kappa$. From (1.9) equation, we have

$$
\sin \varphi^{*}=\frac{\varphi^{\prime}}{\sqrt{\varphi^{\prime 2}+\|W\|^{2}}}, \quad \cos \varphi^{*}=\frac{\|W\|}{\sqrt{\varphi^{\prime 2}+\|W\|^{2}}}, \quad \varphi^{* \prime}=\left(\frac{\varphi^{\prime}}{\sqrt{\varphi^{\prime 2}+\|W\|^{2}}}\right)^{\prime} \frac{\sqrt{\varphi^{\prime 2}+\|W\|^{2}}}{\|W\|} .
$$

Let $\left(\alpha, \alpha^{*}\right)$ be a curve pair in $\mathbb{E}^{3}$. For the vector $C^{*}$ is the direction of the involute curve $\alpha^{*}$ we have

$$
C^{*}=\frac{\sin \varphi\|W\|}{\sqrt{\left(\varphi^{\prime}\right)^{2}+\|W\|^{2}}} T+\frac{\varphi^{\prime}}{\sqrt{\left(\varphi^{\prime}\right)^{2}+\|W\|^{2}}} N+\frac{\cos \varphi\|W\|}{\sqrt{\left(\varphi^{\prime}\right)^{2}+\|W\|^{2}}} B .
$$

where the vector $C$ is the direction of the Darboux vector $W$ of the base curve $\alpha$, [3]. Let $\gamma: I \rightarrow S^{2}$ be a unit speed spherical curve. We denote s as the arc-length parameter of $\gamma$. Let us denote by

$$
\gamma(s)=\gamma(s), \quad t(s)=\gamma^{\prime}(s), d(s)=\gamma(s) \wedge t(s)
$$

$\{\gamma(s), t(s), d(s)\}$ frame is called the Sabban frame of $\gamma$ on $S^{2}$. Then we have the following spherical Frenet formulae of $\gamma$

$$
\gamma^{\prime}(s)=t(s), \quad t^{\prime}(s)=-\gamma(s)+\kappa_{g}(s) d(s), \quad d^{\prime}(s)=-\kappa_{g}(s) t(s)
$$

where $\kappa_{g}$ is called the geodesic curvature of the curve $\gamma$ on $S^{2}$ which is, [11]

$$
\kappa_{g}(s)=\left\langle t^{\prime}(s), d(s)\right\rangle \text {. }
$$




\section{On The Darboux Vector Belonging To Involute Curve A Different View}

In this section, we investigated special Smarandache curves created by Sabban frame $\left\{C^{*}, T_{C^{*}}, C^{*} \wedge T_{C^{*}}\right\}$, that belongs to drawn on the surface of the sphere by the unit Darboux vector of a $\alpha^{*}$ curve are defined. We found some results. These results will be expressed depending on the base curve. Let $\alpha_{C^{*}}\left(s_{C^{*}}\right)=C^{*}\left(s^{*}\right)$ be a unit speed regular spherical curves on $S^{2}$. We denote $s_{C^{*}}$ as the arc-length parameter for drawn on the surface of the sphere by the unit Darboux vector of involute $\left(C^{*}\right)$. Sabban frame for $\left(C^{*}\right)$ is

$$
\alpha_{C^{*}}\left(s_{C^{*}}\right)=C^{*}\left(s^{*}\right)
$$

Differentiating (2.1), we found

$$
T_{C^{*}} \frac{d s_{C^{*}}}{d s^{*}}=\varphi^{* \prime} \cos \varphi^{*} T^{*}-\varphi^{* \prime} \sin \varphi^{*} B^{*}
$$

and we can write

$$
\frac{d s_{C^{*}}}{d s^{*}}=\varphi^{* \prime}
$$

Hence we have

$$
T_{C^{*}}=\cos \varphi^{*} T^{*}-\sin \varphi^{*} B^{*}
$$

and

$$
C^{*} \wedge T_{C^{*}}=N^{*}
$$

From the equation (1.10), we have

$$
\begin{aligned}
C^{*} & =\sin \varphi^{*} T^{*}+\cos \varphi^{*} B^{*} \\
T_{C^{*}} & =\cos \varphi^{*} T^{*}-\sin \varphi^{*} B^{*} \\
C^{*} \wedge T_{C^{*}} & =N^{*} .
\end{aligned}
$$

Then from the equation (1.11) we have the following spherical Frenet formulae of $\left(C^{*}\right)$ is:

$$
\begin{aligned}
C^{* \prime} & =T_{C^{*}} \\
\left(T_{C^{*}}\right)^{\prime} & =-C^{*}+\frac{\left\|W^{*}\right\|}{\varphi^{* \prime}} C^{*} \wedge T_{C^{*}} \\
\left(C^{*} \wedge T_{C^{*}}\right)^{\prime} & =-\frac{\left\|W^{*}\right\|}{\varphi^{* \prime}} T_{C^{*}} .
\end{aligned}
$$

From the equation (1.12), we have the following geodesic curvatures of $\left(C^{*}\right)$ is

$$
\kappa_{g}=\left\langle T_{C^{*}}^{\prime}, C^{*} \wedge T_{C^{*}}\right\rangle \Longrightarrow \kappa_{g}=\frac{\left\|W^{*}\right\|}{\varphi^{* \prime}} .
$$

$\beta_{1}$-Smarandache curve can be defined by

$$
\beta_{1}\left(s_{C^{*}}\right)=\frac{1}{\sqrt{2}}\left(C^{*}+T_{C^{*}}\right)
$$

or substituting the equation (2.4) into equation (2.7), we reach

$$
\beta_{1}\left(s^{*}\right)=\frac{1}{\sqrt{2}}\left(\left(\sin \varphi^{*}+\cos \varphi^{*}\right) T^{*}+\left(\cos \varphi^{*}-\sin \varphi^{*}\right) B^{*}\right) .
$$

Differentiating (2.7), we can write

$$
T_{\beta_{1}}\left(s^{*}\right)=\frac{\varphi^{* \prime}\left(\cos \varphi^{*}-\sin \varphi^{*}\right)}{\sqrt{2 \varphi^{\prime^{\prime}}+\left\|W^{*}\right\|^{2}}} T^{*}+\frac{\left\|W^{*}\right\|}{\sqrt{2 \varphi^{\prime^{\prime}}{ }^{2}+\left\|W^{*}\right\|^{2}}} N^{*}-\frac{\varphi^{* \prime}\left(\cos \varphi^{*}+\sin \varphi^{*}\right)}{\sqrt{2 \varphi^{\prime^{\prime}}+\left\|W^{*}\right\|^{2}}} B^{*} .
$$

Considering the equations (2.8) and (2.9), it easily seen that 


$$
\beta_{1} \wedge T_{\beta_{1}}\left(s^{*}\right)=\frac{\left\|W^{*}\right\|\left(\cos \varphi^{*}+\sin \varphi^{*}\right)}{\sqrt{2\left\|W^{*}\right\|^{2}+4 \varphi^{* \prime 2}}} T^{*}-\frac{\varphi^{* \prime}}{\sqrt{2\left\|W^{*}\right\|^{2}+4 \varphi^{* \prime 2}}} N^{*}+\frac{\left\|W^{*}\right\|\left(\cos \varphi^{*}+\sin \varphi^{*}\right)}{\sqrt{2\left\|W^{*}\right\|^{2}+4 \varphi^{* \prime 2}}} B^{*} .
$$

Differentiating (2.9), where

$$
\left\{\begin{array}{l}
\chi_{1}=-2-\left(\frac{\left\|W^{*}\right\|}{\varphi^{* \prime}}\right)^{2}+\left(\frac{\left\|W^{*}\right\|}{\varphi^{* \prime}}\right)^{\prime}\left(\frac{\left\|W^{*}\right\|}{\varphi^{* \prime}}\right) \\
\chi_{2}=-2-3\left(\frac{\left\|W^{*}\right\|}{\varphi^{* \prime}}\right)^{2}-\left(\frac{\left\|W^{*}\right\|}{\varphi^{* \prime}}\right)^{4}-\left(\frac{\left\|W^{*}\right\|}{\varphi^{* \prime}}\right)^{\prime}\left(\frac{\left\|W^{*}\right\|}{\varphi^{* \prime}}\right) \\
\chi_{3}=2\left(\frac{\left\|W^{*}\right\|}{\varphi^{* \prime}}\right)+\left(\frac{\left\|W^{*}\right\|}{\varphi^{* \prime}}\right)^{3}+\left(\frac{\left\|W^{*}\right\|}{\varphi^{* \prime}}\right)^{\prime}
\end{array}\right.
$$

including we can reach,

$$
T_{\beta_{1}}^{\prime}\left(s^{*}\right)=\frac{\left(\varphi^{* \prime}\right)^{4} \sqrt{2}\left(\chi_{1} \sin \varphi^{*}+\chi_{2} \cos \varphi^{*}\right)}{\left(\left\|W^{*}\right\|^{2}+\left(\varphi^{* \prime}\right)^{2}\right)^{2}} T^{*}+\frac{\chi_{3}\left(\varphi^{* \prime}\right)^{4} \sqrt{2}}{\left(\left\|W^{*}\right\|^{2}+\left(\varphi^{* \prime}\right)^{2}\right)^{2}} N^{*}+\frac{\left(\varphi^{* \prime}\right)^{4} \sqrt{2}\left(\chi_{1} \cos \varphi^{*}-\chi_{2} \sin \varphi^{*}\right)}{\left(\left\|W^{*}\right\|^{2}+\left(\varphi^{* \prime}\right)^{2}\right)^{2}} B^{*} .
$$

From the equation (2.10) and (2.12), $\kappa_{g}^{\beta_{1}}$ geodesic curvature for involute curve $\beta_{1}\left(s^{*}\right)$ is

$$
\begin{aligned}
\kappa_{g}^{\beta_{1}} & =\left\langle T_{\beta_{1}}^{\prime}, \beta_{1} \wedge T_{\beta_{1}}\right\rangle \\
& =\frac{1}{\left(2+\left(\frac{\left\|W^{*}\right\|}{\varphi^{* \prime}}\right)^{2}\right)^{\frac{5}{2}}}\left(\frac{\left\|W^{*}\right\|}{\varphi^{* \prime}} \chi_{1}-\frac{\left\|W^{*}\right\|}{\varphi^{* \prime}} \chi_{2}+2 \chi_{3}\right) .
\end{aligned}
$$

From the equation (1.6) and (1.9), Sabban apparatus of the $\beta_{1}$-Smarandache curve for base curve are

$$
\begin{gathered}
\beta_{1}(s)=\frac{\left(\|W\|-\varphi^{\prime}\right) \sin \varphi}{\sqrt{2 \varphi^{\prime 2}+2\|W\|^{2}}} T-\frac{\varphi^{\prime}+\|W\|}{\sqrt{2 \varphi^{\prime 2}+2\|W\|^{2}}} N+\frac{\left(\|W\|-\varphi^{\prime}\right) \cos \varphi}{\sqrt{2 \varphi^{\prime 2}+2\|W\|^{2}}} B, \\
T_{\beta_{1}}(s)=\frac{\left(-\|W\|-\varphi^{\prime}\right) \eta \sin \varphi-\sqrt{\|W\|^{2}+\varphi^{\prime 2}} \cos \varphi}{\sqrt{\|W\|^{2}+\varphi^{\prime 2}} \sqrt{1+2 \eta^{2}}} T+\frac{\eta\left(\varphi^{\prime}-\|W\|\right)}{\sqrt{\|W\|^{2}+\varphi^{\prime 2}} \sqrt{1+2 \eta^{2}}} N \\
+\frac{\sqrt{\|W\|^{2}+\varphi^{\prime 2}} \sin \varphi-\eta\left(\|W\|+\varphi^{\prime}\right) \cos \varphi}{\sqrt{\|W\|^{2}+\varphi^{\prime 2}} \sqrt{1+2 \eta^{2}}} B, \\
\left(\beta_{1} \wedge T_{\beta_{1}}\right)(s)=\frac{\left(\|W\|+\varphi^{\prime}\right) \sin \varphi-2 \eta \sqrt{\|W\|^{2}+\varphi^{\prime 2}} \cos \varphi}{\sqrt{2+4 \eta^{2}} \sqrt{\|W\|^{2}+\varphi^{\prime 2}}} T-\frac{\varphi^{\prime}-\|W\|}{\sqrt{2+4 \eta^{2}} \sqrt{\|W\|^{2}+\varphi^{\prime 2}}} N \\
\quad+\frac{\left(\|W\|+\varphi^{\prime}\right) \cos \varphi+2 \eta \sqrt{\|W\|^{2}+\varphi^{\prime 2}} \sin \varphi}{\sqrt{2+4 \eta^{2}} \sqrt{\|W\|^{2}+\varphi^{\prime 2}}} B, \\
T_{\beta_{1}}^{\prime}(s)=\frac{\left(\bar{\chi}_{1}\|W\|-\bar{\chi}_{2} \varphi^{\prime}\right) \eta^{4} \sqrt{2} \sin \varphi-\bar{\chi}_{3} \eta^{4} \sqrt{2\|W\|^{2}+2 \varphi^{\prime 2}} \cos \varphi}{\left(1+2 \eta^{2}\right)^{2} \sqrt{\|W\|^{2}+\varphi^{\prime 2}}} T+\frac{\eta^{4} \sqrt{2}\left(\bar{\chi}_{1} \varphi^{\prime}-\bar{\chi}_{2}\|W\|\right)}{\left(1+2 \eta^{2}\right)^{2} \sqrt{\|W\|^{2}+\varphi^{\prime 2}}} N \\
+\frac{\left(\bar{\chi}_{1}\|W\|-\bar{\chi}_{2} \varphi^{\prime}\right) \eta^{4} \sqrt{2} \cos \varphi+\bar{\chi}_{3} \eta^{4} \sqrt{2\|W\|^{2}+2 \varphi^{\prime 2}} \sin \varphi}{\left(1+2 \eta^{2}\right)^{2} \sqrt{\|W\|^{2}+\varphi^{\prime 2}}} B
\end{gathered}
$$

and 


$$
\kappa_{g}^{\beta_{1}}=\frac{1}{\left(2+\frac{1}{\eta^{2}}\right)^{\frac{5}{2}}}\left(\frac{1}{\eta} \bar{\chi}_{1}-\frac{1}{\eta} \bar{\chi}_{2}+2 \bar{\chi}_{3}\right)
$$

where

$$
\frac{1}{\eta}=\frac{\left(\varphi^{*}\right)^{\prime}}{\left\|W^{*}\right\|}=\left(\frac{\varphi^{\prime}}{{\sqrt{\varphi^{\prime 2}+\|W\|^{2}}}^{\prime}}\right)^{\prime} \cos \varphi(c-s)
$$

and

$$
\left\{\begin{array}{l}
\bar{\chi}_{1}=-2-\frac{1}{\eta^{2}}+\frac{1}{\eta^{\prime}} \frac{1}{\eta} \\
\bar{\chi}_{2}=-2-3 \frac{1}{\eta^{2}}-\frac{1}{\eta^{4}}-\frac{1}{\eta^{\prime}} \frac{1}{\eta} \\
\bar{\chi}_{3}=2 \frac{1}{\eta}+\frac{1}{\eta^{3}}+\frac{1}{\eta^{\prime}} .
\end{array}\right.
$$

$\beta_{2}$-Smarandache curve can be defined by

$$
\beta_{2}\left(s_{C^{*}}\right)=\frac{1}{\sqrt{2}}\left(C^{*}+C^{*} \wedge T_{C^{*}}\right)
$$

or from the equation (1.6), (1.8) and (2.4), we can write

$$
\beta_{2}(s)=\frac{\|W\| \sin \varphi-\sqrt{\varphi^{\prime 2}+\|W\|^{2}} \cos \varphi}{\sqrt{2 \varphi^{\prime 2}+2\|W\|^{2}}} T+\frac{\varphi^{\prime}}{\sqrt{2 \varphi^{\prime 2}+2\|W\|^{2}}} N+\frac{\|W\| \cos \varphi+\sqrt{\varphi^{\prime 2}+\|W\|^{2}} \sin \varphi}{\sqrt{2 \varphi^{\prime 2}+2\|W\|^{2}}} B .
$$

Differentiating (2.18), we can write

$$
T_{\beta_{2}}(s)=\frac{-\varphi^{\prime} \sin \varphi}{\sqrt{\|W\|^{2}+\varphi^{\prime 2}}} T+\frac{\|W\|}{\sqrt{\|W\|^{2}+\varphi^{\prime 2}}} N-\frac{\varphi^{\prime} \cos \varphi}{\sqrt{\|W\|^{2}+\varphi^{\prime 2}}} B .
$$

Considering the equations (2.18) and (2.19), with ease seen that

$$
\left(\beta_{2} \wedge T_{\beta_{2}}\right)(s)=\frac{-\|W\| \sin \varphi-\sqrt{\|W\|^{2}+\varphi^{\prime 2}} \cos \varphi}{\sqrt{2\|W\|^{2}+2 \varphi^{\prime 2}}} T-\frac{\varphi^{\prime}}{\sqrt{2\|W\|^{2}+2 \varphi^{\prime 2}}} N+\frac{\sqrt{\|W\|^{2}+\varphi^{\prime 2}} \sin \varphi-\|W\| \cos \varphi}{\sqrt{2\|W\|^{2}+2 \varphi^{\prime 2}}} B .
$$

Differentiating (2.19), we can write

$$
\begin{aligned}
T_{\beta_{2}}^{\prime}(s)= & \frac{-\eta \sqrt{2}\|W\| \sin \varphi-\sqrt{2\|W\|^{2}+2 \varphi^{\prime 2}} \cos \varphi}{(\eta-1) \sqrt{\|W\|^{2}+\varphi^{\prime 2}}} T+\frac{\eta \sqrt{2} \varphi^{\prime}}{(\eta-1) \sqrt{\|W\|^{2}+\varphi^{\prime 2}}} N \\
& +\frac{\sqrt{2\|W\|^{2}+2 \varphi^{\prime 2}} \sin \varphi-\eta \sqrt{2}\|W\| \cos \varphi}{(\eta-1) \sqrt{\|W\|^{2}+\varphi^{\prime 2}}} B .
\end{aligned}
$$

$\kappa_{g}^{\beta_{2}}$ geodesic curvature for base curve $\beta_{2}\left(s_{\beta_{2}}\right)$ is

$$
\kappa_{g}^{\beta_{2}}=\frac{1+\eta}{\eta-1}
$$

$\beta_{3}$-Smarandache curve can be defined by

$$
\beta_{3}\left(s_{C^{*}}\right)=\frac{1}{\sqrt{2}}\left(T_{C^{*}}+C^{*} \wedge T_{C^{*}}\right)
$$

or from the equation (2.4), (1.6) and (1.8), we can write

$$
\beta_{3}(s)=\frac{-\varphi^{\prime} \sin \varphi-\sqrt{\varphi^{\prime 2}+\|W\|^{2}} \cos \varphi}{\sqrt{2 \varphi^{\prime 2}+2\|W\|^{2}}} T+\frac{\|W\|}{\sqrt{2 \varphi^{\prime 2}+2\|W\|^{2}}} N+\frac{\sqrt{\varphi^{\prime 2}+\|W\|^{2}} \sin \varphi-\varphi^{\prime} \cos \varphi}{\sqrt{2 \varphi^{\prime 2}+2\|W\|^{2}}} B .
$$


Differentiating (2.24), we reach

$$
\begin{aligned}
T_{\beta_{3}}(s)= & \frac{\left(\varphi^{\prime}-\eta\|W\|\right) \sin \varphi-\sqrt{\|W\|^{2}+\varphi^{\prime 2}} \cos \varphi}{\sqrt{2+\eta^{2}} \sqrt{\|W\|^{2}+\varphi^{\prime 2}}} T-\frac{\eta \varphi^{\prime}+\|W\|}{\sqrt{2+\eta^{2}} \sqrt{\|W\|^{2}+\varphi^{\prime 2}}} N \\
& +\frac{\left(\varphi^{\prime}-\eta\|W\|\right) \cos \varphi+\sqrt{\|W\|^{2}+\varphi^{\prime 2}} \sin \varphi}{\sqrt{2+\eta^{2}} \sqrt{\|W\|^{2}+\varphi^{\prime 2}}} B .
\end{aligned}
$$

Considering the equations (2.24) and (2.25), it is easily seen

$$
\begin{aligned}
\left(\beta_{3} \wedge T_{\beta_{3}}\right)(s)= & \frac{\left(2\|W\|+\eta \varphi^{\prime}\right) \sin \varphi-\eta \sqrt{\|W\|^{2}+\varphi^{\prime 2}} \cos \varphi}{\sqrt{4+2 \eta^{2}} \sqrt{\|W\|^{2}+\varphi^{\prime 2}}} T+\frac{2 \varphi^{\prime}-\eta\|W\|}{\sqrt{4+2 \eta^{2}} \sqrt{\|W\|^{2}+\varphi^{\prime 2}}} N \\
& +\frac{\left(2\|W\|+\eta \varphi^{\prime}\right) \cos \varphi+\eta \sqrt{\|W\|^{2}+\varphi^{\prime 2}} \sin \varphi}{\sqrt{4+2 \eta^{2}} \sqrt{\|W\|^{2}+\varphi^{\prime 2}}} B .
\end{aligned}
$$

Differentiating (2.25), where

$$
\bar{\sigma}_{1}=\frac{1}{\eta}+2 \frac{1}{\eta^{3}}+2 \frac{1}{\eta^{\prime}} \frac{1}{\eta}, \quad \bar{\sigma}_{2}=-1-3 \frac{1}{\eta^{2}}-2 \frac{1}{\eta^{4}}-\frac{1}{\eta^{\prime}}, \quad \bar{\sigma}_{3}=-\frac{1}{\eta^{2}}-2 \frac{1}{\eta^{4}}+\frac{1}{\eta^{\prime}}
$$

including we have

$$
\begin{aligned}
T_{\beta_{3}}^{\prime}(s)= & \frac{\left(\bar{\sigma}_{2}\|W\|+\bar{\sigma}_{1} \varphi^{\prime}\right) \eta^{4} \sqrt{2} \sin \varphi-\bar{\sigma}_{3} \eta^{4} \sqrt{2\|W\|^{2}+2 \varphi^{\prime 2}} \cos \varphi}{\left(2+\eta^{2}\right)^{2} \sqrt{\varphi^{\prime 2}+\|W\|^{2}}} T+\frac{\left(\bar{\sigma}_{2} \varphi^{\prime}-\bar{\sigma}_{1}\|W\|\right) \eta^{4} \sqrt{2}}{\left(2+\eta^{2}\right)^{2} \sqrt{\varphi^{\prime 2}+\|W\|^{2}}} N \\
& +\frac{\left(\bar{\sigma}_{2}\|W\|+\bar{\sigma}_{1} \varphi^{\prime}\right) \eta^{4} \sqrt{2} \cos \varphi+\bar{\sigma}_{3} \eta^{4} \sqrt{2\|W\|^{2}+2 \varphi^{\prime 2}} \sin \varphi}{\left(2+\eta^{2}\right)^{2} \sqrt{\varphi^{\prime 2}+\|W\|^{2}}} B .
\end{aligned}
$$

$\kappa_{g}^{\beta_{3}}$ geodesic curvature for base curve $\beta_{3}\left(s_{\beta_{3}}\right)$ is

$$
\kappa_{g}^{\beta_{3}}=\frac{1}{\left(2+\frac{1}{\eta^{2}}\right)^{\frac{5}{2}}}\left(2 \frac{1}{\eta} \bar{\sigma}_{1}-\bar{\sigma}_{2}+\bar{\sigma}_{3}\right) .
$$

$\beta_{4}$-Smarandache curve can be defined by

$$
\beta_{4}\left(s_{C^{*}}\right)=\frac{1}{\sqrt{3}}\left(C^{*}+T_{C^{*}}+C^{*} \wedge T_{C^{*}}\right)
$$

or from the equation (1.6), (1.8) and (2.4), we can write

$$
\begin{aligned}
\beta_{4}(s)= & \frac{\left(\|W\|-\varphi^{\prime}\right) \sin \varphi-\sqrt{\varphi^{\prime 2}+\|W\|^{2}} \cos \varphi}{\sqrt{3 \varphi^{\prime 2}+3\|W\|^{2}}} T+\frac{\varphi^{\prime}+\|W\|}{\sqrt{3 \varphi^{\prime 2}+3\|W\|^{2}}} N \\
& +\frac{\left(\|W\|-\varphi^{\prime}\right) \cos \varphi+\sqrt{\varphi^{\prime 2}+\|W\|^{2}} \sin \varphi}{\sqrt{3 \varphi^{\prime 2}+3\|W\|^{2}}} B .
\end{aligned}
$$

Differentiating (2.31), we reach

$$
\begin{aligned}
T_{\beta_{4}}(s)= & \frac{\left((1-\eta) \varphi^{\prime}-\eta\|W\|\right) \sin \varphi-\sqrt{\|W\|^{2}+\varphi^{\prime 2}} \cos \varphi}{\sqrt{2\left(1-\eta+\eta^{2}\right)} \sqrt{\|W\|^{2}+\varphi^{\prime 2}}} T+\frac{(\eta-1)\|W\|-\eta \varphi^{\prime}}{\sqrt{2\left(1-\eta+\eta^{2}\right)} \sqrt{\|W\|^{2}+\varphi^{\prime 2}}} N \\
& +\frac{\left((\eta-1) \varphi^{\prime}-\eta\|W\|\right) \cos \varphi+\sqrt{\|W\|^{2}+\varphi^{\prime 2}} \sin \varphi}{\sqrt{2\left(1-\eta+\eta^{2}\right)} \sqrt{\|W\|^{2}+\varphi^{\prime 2}}} B .
\end{aligned}
$$


Considering the equations (2.31) and (2.32), it is easily seen

$$
\begin{aligned}
\left(\beta_{4} \wedge T_{\beta_{4}}\right)(s)= & \frac{\left((2-\eta)\|W\|+(1+\eta) \varphi^{\prime}\right) \sin \varphi-(2 \eta-1) \sqrt{\|W\|^{2}+\varphi^{\prime 2}} \cos \varphi}{\sqrt{6-6 \eta+6 \eta^{2}} \sqrt{\|W\|^{2}+\varphi^{\prime 2}}} T+\frac{(2-\eta) \varphi^{\prime}-(1+\eta)\|W\|}{\sqrt{6-6 \eta+6 \eta^{2}} \sqrt{\|W\|^{2}+\varphi^{\prime 2}}} N \\
& +\frac{\left((2-\eta)\|W\|+(1+\eta) \varphi^{\prime}\right) \cos \varphi+(2 \eta-1) \sqrt{\|W\|^{2}+\varphi^{\prime 2}} \sin \varphi}{\sqrt{6-6 \eta+6 \eta^{2}} \sqrt{\|W\|^{2}+\varphi^{\prime 2}}} B
\end{aligned}
$$

Differentiating (2.32), where

$$
\left\{\begin{array}{l}
\bar{\rho}_{1}=-2+4 \frac{1}{\eta}-4 \frac{1}{\eta^{2}}+2 \frac{1}{\eta^{3}}+2 \frac{1}{\eta^{\prime}}\left(2 \frac{1}{\eta}-1\right) \\
\bar{\rho}_{2}=-2+2 \frac{1}{\eta}-4 \frac{1}{\eta^{2}}+2 \frac{1}{\eta^{3}}-2 \frac{1}{\eta^{4}}-\frac{1}{\eta^{\prime}}\left(1+\frac{1}{\eta}\right) \\
\bar{\rho}_{3}=2 \frac{1}{\eta}-4 \frac{1}{\eta^{2}}+4 \frac{1}{\eta^{3}}-2 \frac{1}{\eta^{4}}+\frac{1}{\eta^{\prime}}\left(2-\frac{1}{\eta}\right)
\end{array}\right.
$$

including we can write

$$
\begin{aligned}
T_{\beta_{4}}^{\prime}(s)= & \frac{\left(\bar{\rho}_{1}\|W\|-\bar{\rho}_{2} \varphi^{\prime}\right) \eta^{4} \sqrt{3} \sin \varphi-\bar{\rho}_{3} \eta^{4} \sqrt{3\|W\|^{2}+3 \varphi^{\prime 2}} \cos \varphi}{4\left(1-\eta+\eta^{2}\right)^{2} \sqrt{\varphi^{\prime 2}+\|W\|^{2}}} T+\frac{\left(\bar{\rho}_{1} \varphi^{\prime}+\bar{\rho}_{2}\|W\|\right) \eta^{4} \sqrt{3}}{4\left(1-\eta+\eta^{2}\right)^{2} \sqrt{\varphi^{\prime 2}+\|W\|^{2}}} N \\
& +\frac{\left(\bar{\rho}_{1}\|W\|-\bar{\rho}_{2} \varphi^{\prime}\right) \eta^{4} \sqrt{3} \cos \varphi+\bar{\rho}_{3} \sqrt{3\|W\|^{2}+3 \varphi^{\prime 2}} \sin \varphi}{4\left(1-\eta+\eta^{2}\right)^{2} \sqrt{\varphi^{\prime 2}+\|W\|^{2}}} B .
\end{aligned}
$$

$\kappa_{g}^{\beta_{4}}$ geodesic curvature for base curve $\beta_{4}\left(s_{\beta_{4}}\right)$ is

$$
\kappa_{g}^{\beta_{4}}=\frac{\left(2 \frac{1}{\eta}-1\right) \bar{\rho}_{1}+\left(-1-\frac{1}{\eta}\right) \bar{\rho}_{2}+\left(2-\frac{1}{\eta}\right) \bar{\rho}_{3}}{4 \sqrt{2}\left(1-\eta+\eta^{2}\right)^{\frac{5}{2}}} .
$$

Example. Let us consider the unit speed spherical curve:

$$
\alpha(s)=\left\{\frac{2}{5} \sin (2 s)-\frac{1}{40} \sin (8 s),-\frac{2}{5} \cos (2 s)+\frac{1}{40} \cos (8 s), \frac{4}{15} \sin (3 s)\right\}
$$

in the context of definitions, we reach $\left(C^{*}\right)$ curve (see Figure 1) and Smarandache curves according to Sabban frame on $S^{2}$. $\beta_{1}, \beta_{2}, \beta_{3}$ and $\beta_{4}$ (see Figure 2).

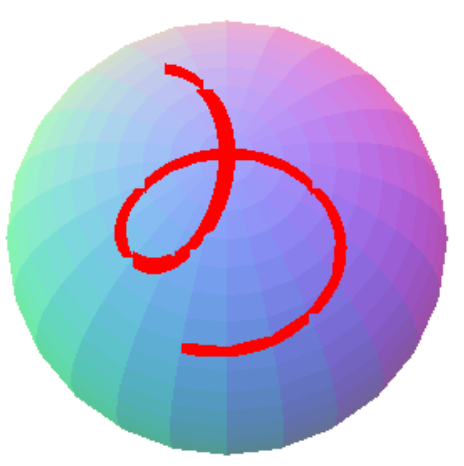

Figure 1. $\left(C^{*}\right)$-curve 


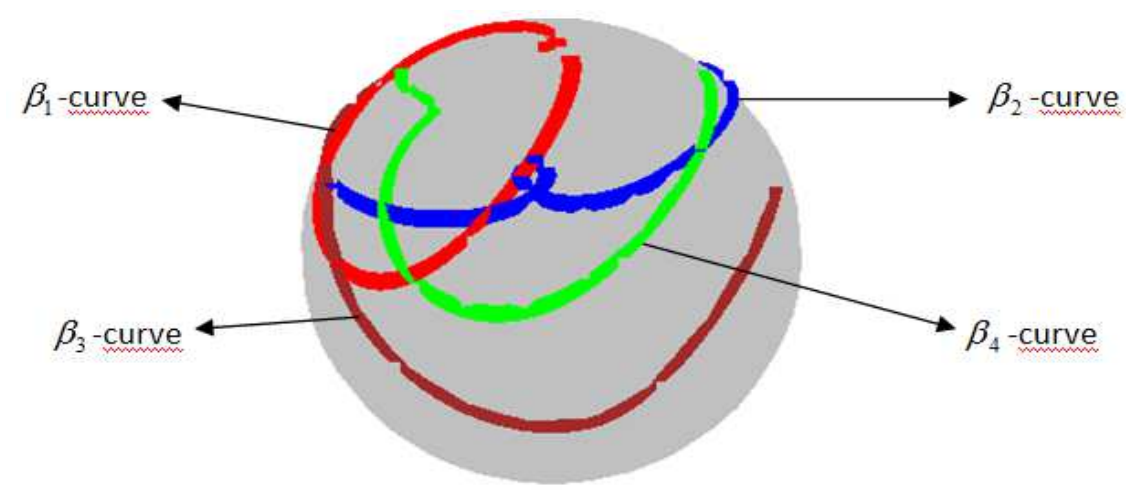

Figure 2. Smarandache curves

\section{References}

[1] Ali A.T., Special Smarandache Curves in the Euclidian Space, International Journal of Mathematical Combinatorics, 2(2010), 30-36.

[2] Bektaş Ö. and Yüce S., Special Smarandache Curves According to Darboux Frame in Euclidean 3-Space, Romanian Journal of Mathematics and Computer sciencel 3(2013), no.1, 48-59.

[3] Bilici M. and Çalıçkan, M., Some Characterizations For The Pair of Involute-evolute curves is Euclidian $E^{3}$, Bulletin of Pure and Applied Sciences, 21E(2002) no.2, 289-294.

[4] Çalıçkan A. and Şenyurt, S., Smarandache Curves In Terms of Sabban Frame of Spherical Indicatrix Curves, Gen. Math. Notes, 31(2015), no.2, 1-15.

[5] Çetin M., Tuncer Y. and Karacan M.K., Smarandache Curves According to Bishop Frame in Euclidean 3-Space, Gen. Math. Notes, 20(2014), 50-66.

[6] Fenchel, W.,On The Differential Geometry of Closed Space Curves, Bulletin of the American Mathematical Society, 57(1951), 44-54.

[7] Hacısalihoğlu H.H., Differantial Geometry(in Turkish), Academic Press Inc. Ankara, 1994.

[8] Şenyurt S. and Sivas S., An Application of Smarandache Curve, University of Ordu Journal of Science and Technology, 3(2013), no.1, 46-60.

[9] Şenyurt S, Altun Y. and Cevahir C., Smarandache Curves According to Sabban Frame of Fixed Pole Curve Belonging to the Bertrand Curves Pair, AIP Conf. Proc. 1726, doi:10.1063/1.4945871, 2016.

[10] Turgut M. and Yilmaz S., Smarandache Curves in Minkowski space-time, International Journal of Mathematical Combinatorics, 3(2008), 51-55.

[11] Taşköprü K. and Tosun M., Smarandache Curves on $S^{2}$, Boletim da Sociedade Paranaense de Matematica 3 Srie. 32(2014), no.1, 51-59.

\section{Affiliations}

SÜLEYMAN ŞENYURT

AdDRESS: Ordu University, Faculty of Arts and Sciences, Department of Mathematics, 52200, Ordu-Turkey. E-MAIL: senyurtsuleyman@hotmail.com

YASIN ALTUN

ADDRESS: Ordu University, Faculty of Arts and Sciences, Department of Mathematics, 52200, Ordu-Turkey. E-MAIL: yasinaltun2852@gmail.com

CEYDA CEVAHIR

ADDRESS: Ordu University, Faculty of Arts and Sciences, Department of Mathematics, 52200, Ordu-Turkey. E-MAIL: Ceydacevahir@gmail.com 